Emir. J. Food Agric. 2009. 21 (1): 10-20

http://cfa.uaeu.ac.ae/ejfa.shtml

\title{
Growth and yield of tomato (Lycopersicon esculentum Mill) as influenced by poultry manure and NPK fertilizer
}

\author{
A. O. Adekiya ${ }^{1}$ and T. M. Agbede ${ }^{2}$ \\ ${ }^{1}$ Department of Crop, Soil and Pest Management, Federal University of Technology, Akure, \\ Nigeria; ${ }^{2}$ Department of Agricultural Engineering Technology, Rufus Giwa Polytechnic, \\ Owo, Ondo State, Nigeria
}

\begin{abstract}
Four field trials were conducted during the years 2006 and 2007 at Owo, the forestsavanna transition zone in southwest Nigeria, to study the effect of poultry manure (PM), NPK 1515-15 fertilizer and NPK 15-15-15 fertilizer + poultry manure on the growth and yield of tomato. Seven treatments were applied to the soil: $0,10,20,30,40 \mathrm{tha}^{-1}$ poultry manure , $300 \mathrm{~kg} \mathrm{ha}^{-1}$ NPK 15 15-15 fertilizer and $150 \mathrm{~kg} \mathrm{ha}^{-1}$ NPK $15-15-15$ fertilizer $+10 \mathrm{tha}^{-1}$ poultry manure. These were laid out in a randomized complete block design and replicated three times. The treatments were compared on the basis of their effect on soil chemical properties, leaf nutrient content, growth and yield of tomato. All levels of poultry manure and NPK 15-15-15 fertilizer + poultry manure increased leaf $\mathrm{N}, \mathrm{P}, \mathrm{K}, \mathrm{Ca}$ and $\mathrm{Mg}$ levels. The soil chemical properties except $\mathrm{pH}$ increased with amount of poultry manure. NPK 15-15-15 fertilizer alone did not increase the soil and leaf $\mathrm{Ca}$ and Mg. All levels of poultry manure, NPK 15-15-15 fertilizer alone and NPK 15-15-15 fertilizer + poultry manure increased the number of leaves, plant height, leaf area, number of fruits and fruit weight significantly. Among poultry manure levels, $30 \mathrm{t} \mathrm{ha}^{-1}$ poultry manure gave the highest fruit yield. Among the seven treatments, NPK 15-15-15 fertilizer + poultry manure gave the highest yield. On an average over the two years, $10,20,30,40 \mathrm{t} \mathrm{ha}^{-1}$ poultry manure, $300 \mathrm{~kg} \mathrm{ha}^{-1} \mathrm{NPK} 15$ 15-15 fertilizer alone and $150 \mathrm{~kg} \mathrm{ha}^{-1}$ NPK $15-15-15$ fertilizer $+10 \mathrm{t} \mathrm{ha}^{-1}$ poultry manure treatments increased fruit weight by $19,36,51,14,20$ and $83 \%$, respectively. Results revealed that poultry manure is a suitable source of nutrients for tomato especially if applied at $30 \mathrm{t} \mathrm{ha}^{-1}$ in the forest-savanna transition zone of southwest Nigeria. The combined use of NPK 15-15-15 fertilizer and poultry manure increased tomato yield compared to the application of NPK 15-15-15 fertilizer or poultry manure alone and is therefore recommended for sustainable productivity. In addition, lesser quantities of poultry manure and NPK 15-15-15 fertilizer would be required, therefore, reducing the amount of money spent on chemical fertilizer.
\end{abstract}

Keywords: Chemical properties, nutrient content, tomato, fruit yield, Nigeria.

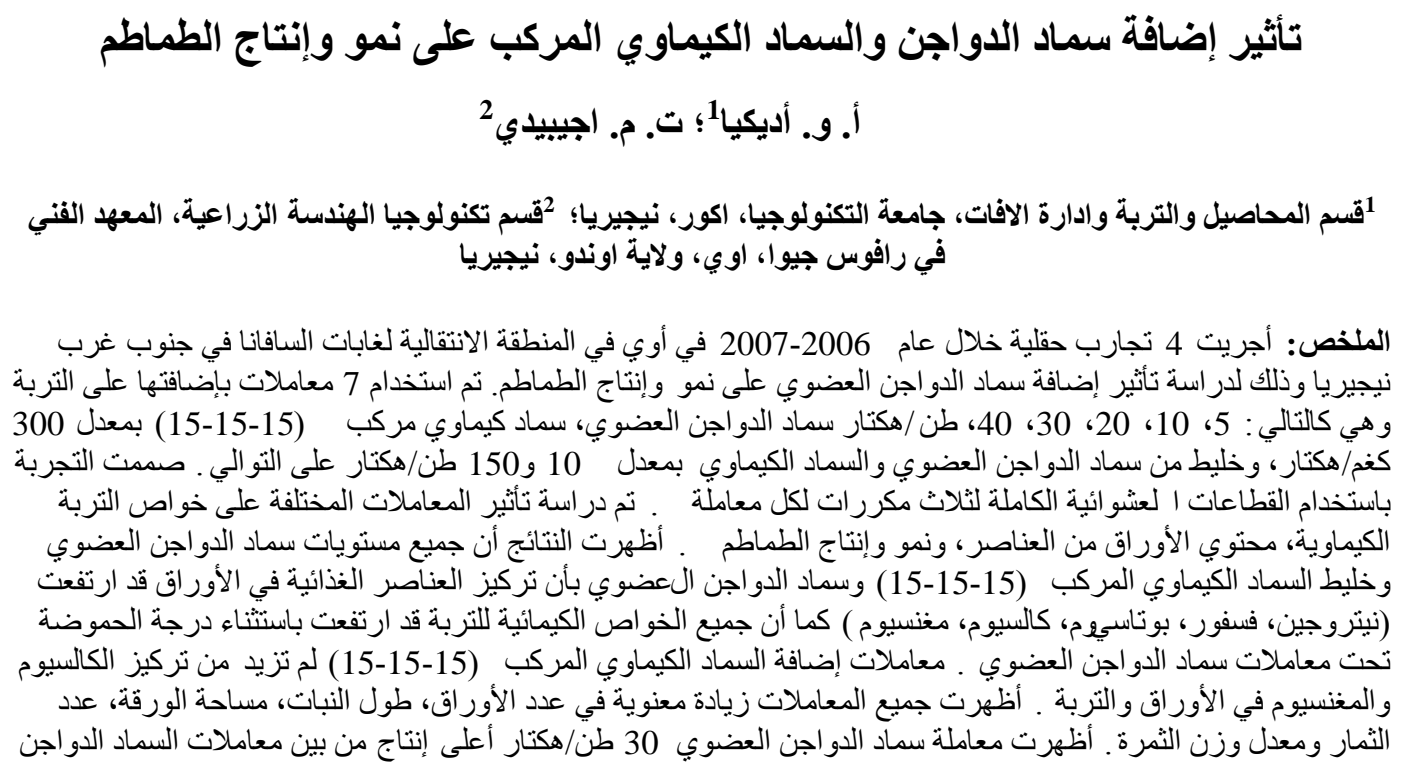




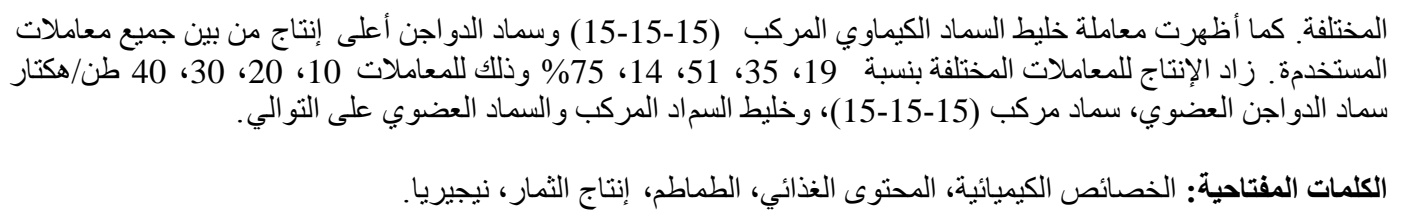

\section{Introduction}

Food is one among the most important basic necessities of man. For Nigeria to meet the millennium development goal in food production, food including tomato must be readily available. Low soil fertility could threaten the security of food production and supply. Soil fertility is a major overriding constraint that affects all aspects of crop production (Mbah, 2006).

In the past years, inorganic fertilizer was advocated for crop production to ameliorate low inherent fertility of soils in the tropics. In addition to being expensive and scarce, the use of inorganic fertilizer has not been helpful in intensive agriculture because it is often associated with reduced crop yield, soil acidity and nutrient imbalance (Ojeniyi, 2000; Ano and Agwu, 2005; Agbede et al., 2008). The need to use renewable forms of energy and reduce costs of fertilizing crops has revived the use of organic fertilizers worldwide (Ayoola and Adeniyan, 2006). Large quantities of organic wastes such as poultry manure are available especially in urban centers and are an effective source of nutrients for vegetables such as tomato (Adediran et al., 2003). Soil fertility status varies considerably with different ecological zones. Infact, even in the same zone, there are micro-differences in soil characteristics. The crop yield response to organic waste is highly variable and depends on the types of wastes, crop type and species, soil type and climate conditions (Adediran et al., 2003).

Akanni and Ojeniyi (2007) carried out a study in the rainforest zone of southwest Nigeria and recommended $20 \mathrm{t} \mathrm{ha}^{-1}$ poultry manure for tomato production. Adediran et al. (2003) also found that poultry manure at $20 \mathrm{tha}^{-1}$ gave the highest tomato yield in the rainforest region of southwestern Nigeria. There is a need to extend this study to the forest-savanna transition zone where there is a different soil type in order to recommend a suitable rate of poultry manure for tomato.

Furthermore, the benefits of using organic materials have not been fully utilized in the humid tropics, partly due to the huge quantities required to satisfy the nutritional needs of crops, transportation and handling costs (Ayoola and Adeniyan, 2006). High and sustained crop yield can be obtained with judicious and balanced NPK fertilization combined with organic matter amendment (Osundare, 2004). In light of these issues, a study was conducted to determine the growth, nutrient content and yield of tomato as influenced by different levels of poultry manure, NPK fertilizer and integrated use of NPK fertilizer and poultry manure in the forest-savanna transition zone of southwest Nigeria.

\section{Materials and Methods}

\section{Site and crop establishment}

Field experiments were conducted during the 2006 and 2007 early and late cropping seasons, respectively at Owo, Nigeria. Owo is located at latitude $5^{0} 12^{\prime} \mathrm{N}$ and longitude $5^{0} 35^{\prime} \mathrm{E}$ within the forestsavanna transition zone of southwest Nigeria. The average annual rainfall varies from 1000-1240 mm. The type of soil is Alfisol classified as Oxic Tropuldalf (USDA) or Luvisol (FAO) derived from quartz, gneiss and schist (Agbede, 2006). There are two rainy seasons, one from March to July and the other from midAugust to November. The farm site was recovered from a four-year fallow after arable cropping. The dominant weeds at the 
site were Siam weed (Chromolaena odorata), Haemorrhage plant (Aspilia africana) and Guinea grass (Panicum maximum). The experiment was laid out on a randomized complete block design (RCBD) with three replications. The treatments were $0,10,20,30,40 \mathrm{t} \mathrm{ha}^{-1}$ poultry manure (PM), $300 \mathrm{~kg} \mathrm{ha}^{-1} \mathrm{NPK} 15$ $15-15$ fertilizer and $150 \mathrm{~kg} \mathrm{ha}^{-1}$ NPK $15-15$ 15 fertilizer $+10 \mathrm{t} \mathrm{ha}^{-1}$ poultry manure (PM). Each plot was $8 \mathrm{~m} \times 8 \mathrm{~m}$, with a $2 \mathrm{~m}$ margin round each plot per plant. The site was manually heaped. Three weeks old local variety of tomato seedlings were transplanted to the field at a spacing of $1 \mathrm{~m}$ $\mathrm{x} 1 \mathrm{~m}$ in April and August of each year (2006 and 2007) for early and late crops, respectively, giving a tomato population of 10,000 plants ha ${ }^{-1}$. The same site was used for the experiments. Poultry manure (PM) and fertilizer were applied by ring method two weeks after transplanting. Two manual weedings were done for each experiment.

\section{Leaf and soil analysis}

During each season, at mid-flowering stage, leaf samples were collected randomly from each plot, oven-dried for $24 \mathrm{hr}$ at $80^{\circ} \mathrm{C}$ and ground in a Willey-mill. These samples were analysed for leaf $\mathrm{N}, \mathrm{P}, \mathrm{K}, \mathrm{Ca}$ and $\mathrm{Mg}$ as described by Tel and Hargarty (1984). Leaf $\mathrm{N}$ was determined by the microKjeldahl digestion method. Ground samples were digested with a nitric-perchloricsulphuric acid mixture (AOAC, 1990). Phosphorus was determined colorimetrically by the vanadomolybdate method, $\mathrm{K}$ was determined using a flame photometer, and $\mathrm{Ca}$ and $\mathrm{Mg}$ were determined by the EDTA titration method.

Before the experiment in 2006, surface soil samples $(0-15 \mathrm{~cm})$ were randomly collected from fifteen different points on the experimental site, bulked together and analysed for physical and chemical properties. After the fruit harvest in 2007, the fertilizer material (poultry manure) used and soil samples were collected randomly from each plot up to a $15 \mathrm{~cm}$ depth from five sampling points per plot. These were analysed for chemical properties as described by Carter (1993). The particle size analysis was done using the Bouyoucos hydrometer method (Sheldrick and Hand Wang, 1993). The organic carbon content in the soil was determined by the dichromate wet oxidation method (Nelson and Sommers, 1996); total N by the microKjeldahl digestion method (Bremner, 1996), and available $\mathrm{P}$ was extracted using Bray-1 solution and determined by molybdenum blue colorimetry (Frank et al., 1998). Exchangeable $\mathrm{K}, \mathrm{Ca}$ and $\mathrm{Mg}$ were extracted using ammonium acetate, $\mathrm{K}$ level was determined using a flame photometer, and $\mathrm{Ca}$ and $\mathrm{Mg}$ by the EDTA titration method (Hendershot and Lalande, 1993). Soil pH was determined by using a soil-water medium at a ratio of $1: 2$ using the digital electronic $\mathrm{pH}$ meter (Ibitoye, 2006).

\section{Determination of growth and yield components}

Ten plants per plot were selected for biweekly determination of plant height and number of leaves. The leaf area was determined at the mid-flowering stage of the tomato plant in each plot. The number and weight of the fruits were evaluated between 72 and 90 days after transplanting.

\section{Statistical analysis}

The data collected were subjected to analysis of variance, and treatment means were compared using Duncan's Multiple Range Test (DMRT) at a 5\% probability level (Steel and Torrie, 1987).

\section{Results}

Properties of the soil prior to experimentation in 2006 and poultry manure used are shown in Table 1 . The soil was sandy loam in texture, low in organic carbon, total $\mathrm{N}$, available $\mathrm{P}$ and exchangeable $\mathrm{Ca}$. The exchangeable $\mathrm{K}$ and $\mathrm{Mg}$ were adequate according to the critical levels of $3.0 \% \mathrm{OM}, 0.20 \% \mathrm{~N}, 10.0 \mathrm{mg} \mathrm{kg}^{-1}$ available $\mathrm{P}, \quad 0.16-0.20 \mathrm{cmol} \quad \mathrm{kg}^{-1}$ exchangeable $\mathrm{K}, \quad 2.0 \mathrm{cmol} \quad \mathrm{kg}^{-1}$ 
exchangeable $\mathrm{Ca}$, and $0.40 \mathrm{cmol} \mathrm{kg}^{-1}$ exchangeable $\mathrm{Mg}$ recommended for crop production in ecological zones of Nigeria (Akinrinde and Obigbesan, 2000). It was found that poultry manure is high in major nutrients required for the growth of fruit crops such as tomato. Application of poultry manure and NPK fertilizer is expected to improve soil fertility and yield of tomato.

Table 2 contains data on the tomato leaf nutrient composition during 2006 and 2007. Compared to $0 \mathrm{t} \mathrm{ha}^{-1}$ poultry manure (control), all levels of poultry manure alone and NPK fertilizer + poultry manure increased leaf $\mathrm{N}, \mathrm{P}, \mathrm{K}, \mathrm{Ca}$ and $\mathrm{Mg}$ amounts significantly. In both years and seasons, the concentration of nutrients increased with the amount of poultry manure up to $40 \mathrm{t} \mathrm{ha}^{-1}$. NPK fertilizer + poultry manure gave higher leaf $\mathrm{N}, \mathrm{P}$ and $\mathrm{K}$ contents than poultry manure alone. NPK fertilizer alone increased leaf $\mathrm{N}, \mathrm{P}$ and $\mathrm{K}$, but did not increase leaf $\mathrm{Ca}$ and $\mathrm{Mg}$. Hence, NPK fertilizer alone gave lower leaf $\mathrm{Ca}$ and $\mathrm{Mg}$ contents as compared to the control.

Data on the chemical composition of the soil at the end of the experiment in 2007 is presented in Table 3. Relative to the control, all levels of poultry manure, NPK fertilizer alone and NPK fertilizer + poultry manure increased the soil $\mathrm{N}, \mathrm{P}$ and $\mathrm{K}$ levels significantly. NPK fertilizer alone did not increase soil $\mathrm{Ca}$ and $\mathrm{Mg}$ significantly. Soil organic carbon (SOC) increased with increasing amounts of poultry manure up to $40 \mathrm{t} \mathrm{ha}^{-1}$. NPK fertilizer alone did not increase SOC. However, soil $\mathrm{pH}$ was reduced from $0 \mathrm{t} \mathrm{ha}^{-1}$ poultry manure to $40 \mathrm{t} \mathrm{ha}^{-1}$ poultry manure. There were no differences in the soil $\mathrm{pH}$ of NPK fertilizer + poultry manure, 10, 20, 30 and 40 t ha $^{-1}$ poultry manure.

Table 1. Soil physical and chemical properties $(0-15 \mathrm{~cm}$ depth) at the experimental site before planting tomato in 2006 and chemical composition of poultry manure used.

\begin{tabular}{lccc}
\hline \multirow{2}{*}{ Soil Property } & $\begin{array}{c}\text { Soil Sample } \\
\text { Value }\end{array}$ & \multicolumn{2}{c}{ Poultry Manure } \\
\cline { 3 - 4 } & & Property & Value \\
\hline Sand $\left(\mathrm{g} \mathrm{kg}^{-1}\right)$ & 675 & PH & 6.8 \\
Silt $\left(\mathrm{g} \mathrm{kg}^{-1}\right)$ & 149 & Organic C $(\%)$ & 14.9 \\
Clay $\left(\mathrm{g} \mathrm{kg}^{-1}\right)$ & 176 & Nitrogen $(\%)$ & 2.23 \\
Textural class & Sandy loam & C:N & 6.7 \\
pH $($ Water $)$ & 5.9 & Phosphorus (\%) & 0.83 \\
Organic carbon $\left(\mathrm{g} \mathrm{kg}^{-1}\right)$ & 1.47 & Potassium (\%) & 2.35 \\
Nitrogen $\left(\mathrm{g} \mathrm{kg}^{-1}\right)$ & 0.18 & Calcium (\%) & 1.42 \\
Phosphorus $\left(\mathrm{mg} \mathrm{kg}^{-1}\right)$ & 9.8 & Magnesium (\%) & 0.58 \\
Potassium $\left(\mathrm{cmol} \mathrm{kg}^{-1}\right)$ & 0.95 & & \\
Calcium $\left(\mathrm{cmol} \mathrm{kg}^{-1}\right)$ & 1.0 & & \\
Magnesium $\left(\mathrm{cmol} \mathrm{kg}^{-1}\right)$ & 0.96 & & \\
\hline
\end{tabular}


Emir. J. Food Agric. 2009. 21 (1): 10-20

http://cfa.uaeu.ac.ae/ejfa.shtml

Table 2. Effect of poultry manure (PM), NPK 15-15-15 fertilizer and their combination on leaf nutrient composition of tomato crop in 2006 and 2007.

\begin{tabular}{|c|c|c|c|c|c|c|c|c|c|c|c|c|c|c|c|c|c|c|c|c|}
\hline \multirow{3}{*}{ Treatments } & \multicolumn{4}{|c|}{$N\left(g 1_{100 g^{-1}}\right)$} & \multicolumn{4}{|c|}{$P\left(g 100 g^{-1}\right)$} & \multicolumn{4}{|c|}{$K\left(g \mid 100 g^{-1}\right)$} & \multicolumn{4}{|c|}{$\mathrm{Ca}\left(\mathrm{g} 100 \mathrm{~g}^{-1}\right)$} & \multicolumn{4}{|c|}{$\operatorname{Mg}\left(\operatorname{g~} 100 g^{-1}\right)$} \\
\hline & \multicolumn{2}{|c|}{2006} & \multicolumn{2}{|c|}{2007} & \multicolumn{2}{|c|}{2006} & \multicolumn{2}{|c|}{2007} & \multicolumn{2}{|c|}{2006} & \multicolumn{2}{|c|}{2007} & \multicolumn{2}{|c|}{2006} & \multicolumn{2}{|c|}{2007} & \multicolumn{2}{|c|}{2006} & \multicolumn{2}{|c|}{2007} \\
\hline & ${ }^{\mathrm{a}} \mathbf{E}$ & ${ }^{b} \mathbf{L}$ & ${ }^{\mathrm{a}} \mathbf{E}$ & ${ }^{b} \mathbf{L}$ & ${ }^{\mathrm{a}} \mathbf{E}$ & ${ }^{b} \mathbf{L}$ & ${ }^{\mathrm{a}} \mathbf{E}$ & ${ }^{b} \mathbf{L}$ & ${ }^{\mathrm{a}} \mathbf{E}$ & ${ }^{\mathbf{b}} \mathbf{L}$ & ${ }^{\mathrm{a}} \mathbf{E}$ & ${ }^{\mathbf{b}} \mathbf{L}$ & ${ }^{\mathrm{a}} \mathbf{E}$ & ${ }^{b} \mathbf{L}$ & ${ }^{\mathrm{a}} \mathbf{E}$ & ${ }^{b} \mathbf{L}$ & ${ }^{\mathrm{a}} \mathbf{E}$ & ${ }^{\mathbf{b}} \mathbf{L}$ & ${ }^{\mathrm{a}} \mathbf{E}$ & ${ }^{b} \mathbf{L}$ \\
\hline $0 \mathrm{t} \mathrm{ha}^{-1} \mathrm{PM}$ & $1.72 \mathrm{~g}$ & $1.60 \mathrm{~g}$ & $1.63 \mathrm{f}$ & $1.78 \mathrm{f}$ & $0.28 \mathrm{e}$ & $0.21 f$ & $0.25 f$ & $0.27 \mathrm{f}$ & $1.71 \mathrm{e}$ & $1.60 \mathrm{e}$ & $1.50 \mathrm{f}$ & $1.66 f$ & $0.18 \mathrm{~d}$ & $0.20 \mathrm{~d}$ & $0.25 \mathrm{e}$ & $0.23 f$ & $0.30 \mathrm{e}$ & $0.40 \mathrm{~d}$ & $0.42 \mathrm{f}$ & $0.45 f$ \\
\hline $10 \mathrm{t} \mathrm{ha}^{-1} \mathrm{PM}$ & $1.90 \mathrm{f}$ & $1.90 \mathrm{f}$ & $3.20 \mathrm{e}$ & $3.40 \mathrm{e}$ & $0.34 \mathrm{~d}$ & $0.31 \mathrm{e}$ & $0.32 \mathrm{e}$ & $0.34 \mathrm{e}$ & $1.89 \mathrm{~d}$ & $1.99 \mathrm{~d}$ & $1.79 \mathrm{e}$ & $1.98 \mathrm{de}$ & $0.36 \mathrm{c}$ & $0.38 \mathrm{c}$ & $0.40 \mathrm{~d}$ & $0.38 \mathrm{e}$ & $0.51 d$ & $0.70 \mathrm{c}$ & $0.77 \mathrm{~cd}$ & $0.76 \mathrm{~d}$ \\
\hline $20 \mathrm{tha}^{-1} \mathrm{PM}$ & $3.40 \mathrm{~d}$ & $3.10 \mathrm{e}$ & $3.95 \mathrm{~d}$ & $4.20 \mathrm{~d}$ & $0.41 \mathrm{c}$ & $0.46 \mathrm{~cd}$ & $0.40 \mathrm{~d}$ & $0.42 \mathrm{~cd}$ & $2.05 \mathrm{~cd}$ & $2.60 \mathrm{c}$ & $1.98 \mathrm{~cd}$ & $2.20 \mathrm{c}$ & $0.48 b$ & $0.49 b$ & $0.56 \mathrm{c}$ & $0.50 \mathrm{c}$ & $0.58 \mathrm{c}$ & $0.72 \mathrm{c}$ & $0.81 \mathrm{c}$ & $0.85 \mathrm{c}$ \\
\hline $30 \mathrm{t} \mathrm{ha}^{-1} \mathrm{PM}$ & $4.20 c$ & $4.40 \mathrm{c}$ & $4.50 \mathrm{c}$ & $4.95 \mathrm{~b}$ & $0.50 \mathrm{~b}$ & $0.55 \mathrm{~b}$ & $0.56 b$ & $0.49 \mathrm{~b}$ & $2.60 \mathrm{~b}$ & $3.20 \mathrm{~b}$ & $2.70 \mathrm{~b}$ & $2.72 b$ & $0.60 \mathrm{a}$ & $0.65 \mathrm{a}$ & $0.78 \mathrm{a}$ & $0.70 \mathrm{a}$ & $0.80 \mathrm{a}$ & $0.89 \mathrm{a}$ & $0.98 \mathrm{a}$ & $0.99 \mathrm{a}$ \\
\hline $40 \mathrm{t} \mathrm{ha}^{-1} \mathrm{PM}$ & $4.50 \mathrm{bc}$ & $4.70 \mathrm{bc}$ & $5.20 \mathrm{~b}$ & $5.50 \mathrm{a}$ & $0.40 \mathrm{c}$ & $0.43 \mathrm{~d}$ & $0.42 \mathrm{~d}$ & $0.39 \mathrm{~d}$ & $1.91 d$ & $1.67 \mathrm{e}$ & $1.60 \mathrm{f}$ & $1.99 \mathrm{de}$ & $0.60 \mathrm{a}$ & $0.65 \mathrm{a}$ & $0.68 \mathrm{~b}$ & $0.60 \mathrm{~b}$ & $0.67 \mathrm{~b}$ & $0.81 \mathrm{~b}$ & $0.90 \mathrm{~b}$ & $0.90 \mathrm{bc}$ \\
\hline $\begin{array}{l}\text { NPK }\left(300 \mathrm{~kg} \mathrm{ha}^{-1}\right) \\
\text { fertilizer }\end{array}$ & $3.00 \mathrm{e}$ & $3.50 \mathrm{~d}$ & $3.96 \mathrm{~d}$ & $4.41 \mathrm{~cd}$ & $0.41 \mathrm{c}$ & $0.45 \mathrm{~cd}$ & $0.46 \mathrm{~cd}$ & $0.40 \mathrm{~d}$ & $1.91 d$ & $2.02 \mathrm{~d}$ & $1.90 \mathrm{de}$ & $1.85 \mathrm{e}$ & $0.17 \mathrm{~d}$ & $0.20 \mathrm{~d}$ & $0.23 \mathrm{e}$ & $0.22 \mathrm{f}$ & $0.31 \mathrm{e}$ & $0.39 \mathrm{~d}$ & $0.43 \mathrm{f}$ & $0.45 f$ \\
\hline $\begin{array}{l}\text { NPK }\left(150 \mathrm{~kg} \mathrm{ha}^{-1}\right) \\
\text { fertilizer+10 } \mathrm{t} \mathrm{ha}^{-1} \\
\text { PM }\end{array}$ & $5.10 \mathrm{a}$ & $5.30 \mathrm{a}$ & $5.80 \mathrm{a}$ & $5.60 \mathrm{a}$ & $0.57 \mathrm{a}$ & $0.63 \mathrm{a}$ & $0.65 \mathrm{a}$ & $0.55 \mathrm{a}$ & $3.10 \mathrm{a}$ & $3.90 \mathrm{a}$ & $3.50 \mathrm{a}$ & $2.98 \mathrm{a}$ & $0.36 \mathrm{c}$ & $0.39 \mathrm{c}$ & $0.40 \mathrm{~d}$ & $0.42 \mathrm{~d}$ & $0.53 \mathrm{~d}$ & $0.71 \mathrm{c}$ & 0.71de & $0.66 \mathrm{e}$ \\
\hline
\end{tabular}


A. O. Adekiya and T. M. Agbede

Table 3. Effect of poultry manure (PM), NPK fertilizer 15-15-15 and their combination on soil nutrient composition at the end of the experiment in 2007.

\begin{tabular}{|c|c|c|c|c|c|c|c|}
\hline Treatments & $\begin{array}{c}\mathbf{p H} \\
\left(\mathrm{H}_{2} \mathrm{O}\right)\end{array}$ & $\begin{array}{l}\text { Organic } \\
\text { carbon } \\
\left(\mathrm{g} \mathrm{kg}^{-1}\right)\end{array}$ & $\begin{array}{c}\text { Nitrogen } \\
\left(\mathrm{g} \mathrm{kg}^{-1}\right)\end{array}$ & $\begin{array}{c}\text { Phosphoru } \\
\left(\mathrm{mg} \mathrm{kg}^{-1}\right)\end{array}$ & $\begin{array}{l}\text { Potassium } \\
\left(\mathrm{cmol} \mathrm{kg}^{-1}\right)\end{array}$ & $\begin{array}{l}\text { Calcium } \\
\left(\mathrm{cmol} \mathrm{kg}^{-1}\right)\end{array}$ & $\begin{array}{c}\text { Magnesium } \\
\left(\mathrm{cmol} \mathrm{kg}^{-1}\right)\end{array}$ \\
\hline 0 tha $^{-1} \mathrm{PM}$ & $6.98 \mathrm{a}$ & $1.30 \mathrm{~d}$ & $0.14 \mathrm{f}$ & $8.3 e$ & $0.80 \mathrm{~d}$ & $0.40 \mathrm{~d}$ & $0.81 \mathrm{e}$ \\
\hline 10 tha $^{-1} \mathrm{PM}$ & 6.60ab & $2.20 \mathrm{c}$ & $0.22 \mathrm{e}$ & 20.1d & $1.02 \mathrm{c}$ & $0.94 c$ & $0.93 \mathrm{~d}$ \\
\hline 20 tha $^{-1} \mathrm{PM}$ & $6.49 \mathrm{ab}$ & $2.50 \mathrm{~b}$ & $0.35 \mathrm{~d}$ & $30.0 \mathrm{c}$ & $1.56 \mathrm{~b}$ & $1.05 \mathrm{~b}$ & $1.30 \mathrm{~b}$ \\
\hline 30 tha $^{-1} \mathrm{PM}$ & 6.39ab & $2.72 \mathrm{a}$ & $0.48 \mathrm{c}$ & $42.2 \mathrm{a}$ & $1.90 \mathrm{a}$ & $1.46 \mathrm{a}$ & $1.65 \mathrm{a}$ \\
\hline 40 tha-1 PM & $6.25 \mathrm{bc}$ & $2.79 a$ & $0.54 b$ & $43.4 \mathrm{a}$ & $1.98 \mathrm{a}$ & $1.40 \mathrm{a}$ & $1.60 \mathrm{a}$ \\
\hline $\begin{array}{l}\text { NPK }\left(300 \mathrm{~kg} \mathrm{ha}^{-1}\right) \\
\text { Fertilizer }\end{array}$ & $6.10 \mathrm{bcd}$ & $1.00 \mathrm{e}$ & $0.35 \mathrm{~d}$ & $37.2 b$ & $1.02 \mathrm{c}$ & $0.39 d$ & $0.79 \mathrm{e}$ \\
\hline $\begin{array}{l}\text { NPK }\left(150 \mathrm{~kg} \mathrm{ha}^{-1}\right) \\
\text { fertilizer }+10 \mathrm{tha}^{-1} \mathrm{PM}\end{array}$ & $6.55 \mathrm{ab}$ & $2.21 \mathrm{c}$ & $0.60 \mathrm{a}$ & $43.9 \mathrm{a}$ & $1.90 \mathrm{a}$ & $0.95 \mathrm{c}$ & $1.35 b c$ \\
\hline
\end{tabular}

Values followed by the same letter(s) in a column are not significantly different at $\mathrm{p}=0.05$ according to

Duncan's multiple range test (DMRT).

Data on the growth and yield of tomato are presented in Tables 4 and 5, respectively. Poultry manure, NPK fertilizer alone and NPK fertilizer + poultry manure significantly increased plant height, number of leaves and leaf area as compared to the control. NPK fertilizer + poultry manure mostly enhanced the growth parameters. Growth parameters during both years increased with the amount of poultry manure. $30 \mathrm{t} \mathrm{ha}^{-1}$ poultry manure gave the highest growth parameters. Values of these parameters reduced beyond $30 \mathrm{t} \mathrm{ha}^{-1}$ poultry manure. The numbers of fruits per plant and fruit weight (Table 5) increased with poultry manure alone and NPK fertilizer + poultry manure applications. The mean fruit weight $\left(\mathrm{t} \mathrm{ha}^{-1}\right)$ for $0,10,20,30,40 \mathrm{t} \mathrm{ha}^{-1}$ poultry manure applications, $300 \mathrm{~kg} \mathrm{ha}^{-1}$ NPK fertilizer alone and $150 \mathrm{~kg} \mathrm{ha}^{-1}$ NPK fertilizer $+10 \mathrm{t} \mathrm{ha}^{-1}$ poultry manure in 2006 were $9.0,10.2,11.7,13.0,10.1,10.1$ and $14.9 \mathrm{t} \mathrm{ha}^{-1}$, respectively. While in 2007 , they were $7.9,9.9,11.1,12.5,9.1,10.1$ and 16.0 $\mathrm{t} \mathrm{ha}^{-1}$, respectively. Therefore, $10,20,30,40$ $\mathrm{t} \mathrm{ha}{ }^{-1}$ poultry manure, $300 \mathrm{~kg} \mathrm{ha}^{-1}$ NPK fertilizer alone and $150 \mathrm{~kg} \mathrm{ha}^{-1}$ NPK fertilizer $+10 \mathrm{t} \mathrm{ha}^{-1}$ poultry manure increased fruit weight by 13.3, 30.0, 44.4, 12.2, 12.2 and 65.6, respectively in 2006; and 25.3, 40.5, 58.2, 15.2, 27.8 and 102.5, respectively in 2007. The corresponding increases in the number of fruits per plant were $37.5,37.5,65.6,25.0,25.0$ and 93.8, respectively in 2006; and 36.4, 39.4, 75.8, 21.2, 21.2 and 93.9, respectively in 2007 . During both years, among poultry manure levels, $30 \mathrm{t} \mathrm{ha}^{-1}$ poultry manure gave the highest fruit yield after which the amount decreased with the use of $40 \mathrm{t} \mathrm{ha}^{-1}$ poultry manure. All levels of poultry manure outyielded NPK fertilizer alone except in 2007 where NPK alone out-yielded the $40 \mathrm{t} \mathrm{ha}^{-1}$ poultry manure level. NPK fertilizer + poultry manure mostly enhanced fruit yield in both years. 
Emir. J. Food Agric. 2009. 21 (1): 10-20

http://cfa.uaeu.ac.ae/ejfa.shtml

Table 4. Effect of poultry manure (PM), NPK 15-15-15 fertilizer and their combination on the growth of tomato crop in 2006 and 2007.

\begin{tabular}{|c|c|c|c|c|c|c|c|c|c|c|c|c|}
\hline \multirow{3}{*}{ Treatments } & \multicolumn{4}{|c|}{ Plant height (m) } & \multicolumn{4}{|c|}{ Number of leaves/plant } & \multicolumn{4}{|c|}{ Leaf area $\left(\mathbf{m}^{2}\right)$} \\
\hline & \multicolumn{2}{|c|}{2006} & \multicolumn{2}{|c|}{2007} & \multicolumn{2}{|c|}{2006} & \multicolumn{2}{|c|}{2007} & \multicolumn{2}{|c|}{2006} & \multicolumn{2}{|c|}{2007} \\
\hline & ${ }^{\mathrm{a}} \mathbf{E}$ & ${ }^{\mathbf{b}} \mathbf{L}$ & $\mathbf{E}$ & $\mathbf{L}$ & $\mathbf{E}$ & $\mathbf{L}$ & $\mathbf{E}$ & $\mathbf{L}$ & $\mathbf{E}$ & $\mathbf{L}$ & $\mathbf{E}$ & $\mathbf{L}$ \\
\hline $0 \mathrm{t} \mathrm{ha}^{-1} \mathrm{PM}$ & $0.65 \mathrm{e}$ & $0.69 \mathrm{e}$ & $0.50 \mathrm{f}$ & $0.55 \mathrm{e}$ & $29 \mathrm{~g}$ & $26 \mathrm{~g}$ & $31 \mathrm{~g}$ & $28 \mathrm{~g}$ & $0.17 \mathrm{f}$ & $0.21 \mathrm{e}$ & $0.20 \mathrm{f}$ & $0.25 \mathrm{e}$ \\
\hline $10 \mathrm{tha}^{-1} \mathrm{PM}$ & $0.77 \mathrm{~d}$ & $0.79 \mathrm{~d}$ & $0.61 \mathrm{e}$ & $0.68 \mathrm{~d}$ & $44 \mathrm{f}$ & $40 \mathrm{f}$ & $48 f$ & $43 \mathrm{f}$ & $0.19 \mathrm{e}$ & $0.35 \mathrm{~d}$ & $0.26 \mathrm{~d}$ & $0.30 \mathrm{~d}$ \\
\hline $20 \mathrm{t} \mathrm{ha}^{-1} \mathrm{PM}$ & $0.88 \mathrm{c}$ & $0.91 \mathrm{c}$ & $0.78 \mathrm{c}$ & $0.83 \mathrm{c}$ & $79 \mathrm{c}$ & $68 \mathrm{c}$ & $78 \mathrm{c}$ & $79 \mathrm{c}$ & $0.25 \mathrm{c}$ & $0.49 b$ & $0.29 \mathrm{c}$ & $0.37 \mathrm{c}$ \\
\hline $30 \mathrm{t} \mathrm{ha}^{-1} \mathrm{PM}$ & $0.98 b$ & $1.01 \mathrm{~b}$ & $0.88 b$ & $0.93 b$ & $93 b$ & $99 \mathrm{~b}$ & $103 b$ & $92 b$ & $0.28 b$ & $0.68 \mathrm{a}$ & $0.37 \mathrm{~b}$ & $0.45 \mathrm{~b}$ \\
\hline $40 \mathrm{tha}^{-1} \mathrm{PM}$ & $0.78 \mathrm{~d}$ & $0.80 \mathrm{~d}$ & $0.69 \mathrm{~d}$ & $0.73 d$ & $59 \mathrm{~d}$ & $50 \mathrm{e}$ & $59 \mathrm{e}$ & $56 \mathrm{~d}$ & $0.22 \mathrm{~d}$ & $0.39 \mathrm{c}$ & $0.23 \mathrm{e}$ & $0.30 \mathrm{~d}$ \\
\hline $\begin{array}{l}\text { NPK }\left(300 \mathrm{~kg} \mathrm{ha}^{-1}\right) \\
\text { Fertilizer }\end{array}$ & $0.79 \mathrm{~d}$ & $0.82 \mathrm{~d}$ & $0.67 \mathrm{~d}$ & $0.70 \mathrm{~d}$ & $48 \mathrm{e}$ & $60 d$ & $68 \mathrm{~d}$ & $47 \mathrm{e}$ & $0.20 \mathrm{e}$ & $0.40 \mathrm{c}$ & $0.30 \mathrm{c}$ & $0.31 d$ \\
\hline $\begin{array}{l}\text { NPK }\left(150 \mathrm{~kg} \mathrm{ha}^{-1}\right) \\
\text { fertilizer }+10 \mathrm{tha}^{-1} \mathrm{PM}\end{array}$ & $1.08 \mathrm{a}$ & $1.12 \mathrm{a}$ & $0.99 a$ & $1.03 \mathrm{a}$ & $105 a$ & $115 \mathrm{a}$ & $120 \mathrm{a}$ & $102 \mathrm{a}$ & $0.31 \mathrm{a}$ & $0.69 \mathrm{a}$ & $0.42 \mathrm{a}$ & $0.50 \mathrm{a}$ \\
\hline
\end{tabular}

Values followed by the same letter(s) in a column are not significantly different at

$\mathrm{p}=0.05$ according to Duncan's multiple range test (DMRT)

${ }^{\mathrm{a}} \mathrm{E}=$ Early season tomato crop

${ }^{b} \mathrm{~L}=$ Late season tomato crop

Table 5. Effect of poultry manure (PM), NPK 15-15-15 fertilizer and their combination on the yield of tomato crop in 2006 and 2007.

\begin{tabular}{lcccccccc}
\hline \multirow{2}{*}{ Treatments } & \multicolumn{3}{c}{ Fruit weight $\left(\mathbf{t ~ h a}^{-1}\right)$} & \multicolumn{3}{c}{ Number of fruits per plant } \\
& \multicolumn{2}{c}{$\mathbf{2 0 0 6}$} & \multicolumn{2}{c}{$\mathbf{2 0 0 7}$} & \multicolumn{2}{c}{$\mathbf{2 0 0 6}$} & \multicolumn{2}{c}{$\mathbf{2 0 0 7}$} \\
\cline { 2 - 9 } & ${ }^{\mathrm{a}} \mathbf{E}$ & ${ }^{\mathrm{b}} \mathbf{L}$ & $\mathbf{E}$ & $\mathbf{L}$ & $\mathbf{E}$ & $\mathbf{L}$ & $\mathbf{E}$ & $\mathbf{L}$ \\
\hline $0 \mathrm{t} \mathrm{ha}^{-1} \mathrm{PM}$ & $8.9 \mathrm{e}$ & $9.1 \mathrm{e}$ & $7.7 \mathrm{f}$ & $8.0 \mathrm{f}$ & $15 \mathrm{~d}$ & $17 \mathrm{e}$ & $16 \mathrm{e}$ & $17 \mathrm{f}$ \\
$10 \mathrm{t} \mathrm{ha}^{-1} \mathrm{PM}$ & $10.0 \mathrm{~d}$ & $10.3 \mathrm{~d}$ & $9.6 \mathrm{de}$ & $10.1 \mathrm{~d}$ & $20 \mathrm{c}$ & $24 \mathrm{c}$ & $20 \mathrm{~d}$ & $25 \mathrm{c}$ \\
$20 \mathrm{tha}^{-1} \mathrm{PM}$ & $11.5 \mathrm{c}$ & $11.9 \mathrm{c}$ & $10.9 \mathrm{c}$ & $11.3 \mathrm{c}$ & $20 \mathrm{c}$ & $24 \mathrm{c}$ & $21 \mathrm{~cd}$ & $25 \mathrm{c}$ \\
$30 \mathrm{t} \mathrm{ha}^{-1} \mathrm{PM}$ & $12.9 \mathrm{~b}$ & $13.0 \mathrm{~b}$ & $12.3 \mathrm{~b}$ & $12.7 \mathrm{~b}$ & $22 \mathrm{~b}$ & $31 \mathrm{~b}$ & $23 \mathrm{ab}$ & $35 \mathrm{~b}$ \\
$40 \mathrm{t} \mathrm{ha}^{-1} \mathrm{PM}$ & $10.2 \mathrm{~d}$ & $10.0 \mathrm{~d}$ & $9.0 \mathrm{e}$ & $9.2 \mathrm{e}$ & $20 \mathrm{c}$ & $20 \mathrm{~d}$ & $16 \mathrm{e}$ & $24 \mathrm{~cd}$ \\
$\mathrm{NPK}\left(300 \mathrm{~kg} \mathrm{ha}^{-1}\right)$ fertilizer & $10.0 \mathrm{~d}$ & $10.3 \mathrm{~d}$ & $9.8 \mathrm{de}$ & $10.3 \mathrm{~d}$ & $20 \mathrm{c}$ & $20 \mathrm{~d}$ & $17 \mathrm{ef}$ & $23 \mathrm{de}$ \\
$\mathrm{NPK}\left(150 \mathrm{~kg} \mathrm{ha}^{-1}\right)$ fertilizer & $14.8 \mathrm{a}$ & $14.9 \mathrm{a}$ & $17.2 \mathrm{a}$ & $14.7 \mathrm{a}$ & $25 \mathrm{a}$ & $37 \mathrm{a}$ & $24 \mathrm{a}$ & $40 \mathrm{a}$ \\
$+10 \mathrm{t} \mathrm{ha}^{-1} \mathrm{PM}$ & & & & & & & & \\
\hline
\end{tabular}

Values followed by the same letter(s) in a column are not significantly different at

$\mathrm{p}=0.05$ according to Duncan's multiple range test (DMRT)

${ }^{\mathrm{a}} \mathrm{E}=$ Early season tomato crop

${ }^{\mathrm{b}} \mathrm{L}=$ Late season tomato crop 


\section{Discussion}

The increase of $\mathrm{N}, \mathrm{P}, \mathrm{K}, \mathrm{Ca}$ and $\mathrm{Mg}$ contents of tomato due to the application of poultry manure is consistent with the use of poultry manure as fertilizer for tomato production (Agele et al., 2001; Akanni, 2005). It was found that $30 \mathrm{t} \mathrm{ha}^{-1}$ poultry manure gave the most growth and highest fruit yield among all poultry manure levels, although leaf $\mathrm{N}$ increased with amount of poultry manure up to $40 \mathrm{t} \mathrm{ha}^{-1}$ poultry manure. Olasantan (1991) also found that fruit yield of the tomato plant was reduced at higher $\mathrm{N}$ application rates. It was suggested that there was a nutrient imbalance in tomato with a large increase in $\mathrm{N}$ supplied from poultry manure. Reduction in growth and yield when more than $30 \mathrm{tha}$ 1 poultry manure was used could also be adduced to soil acidity. Soil $\mathrm{pH}$ tended to reduce with a rise in the amount of poultry manure suggesting that poultry manure lead to increased acidity in the soil. Excess $\mathrm{N}$ in the soil and soil acidity could cause nutrient imbalance in the tomato crop and a reduction in the uptake of certain nutrients (Ewulo et al., 2008). The finding that all levels of poultry manure performed better than the NPK fertilizer alone was adduced to the fact that poultry manure supply more nutrients than NPK fertilizer. The poultry manure could have supplied micronutrients which are essential for tomato growth and yield. Stephenson et al. (1990) and Oladotun (2002) reported that poultry manure contains macro and micro nutrients such as N, P, K, S, Ca, Mg, Cu, Mn, Zn, Bo and Fe. Agele (2001) also found that poultry manure litters resulted in better growth and yield of tomato than NPK fertilizer alone. In this study, tomato performed best in terms of growth and yield under NPK fertilizer + poultry manure treatment in both years. This could be attributed to increased nutrient use efficiency, following the inclusion of the NPK fertilizer. As recorded for the treatment, the highest tomato fruit yield recorded for this treatment is consistent with the maximum presence of $\mathrm{N}, \mathrm{P}, \mathrm{K}, \mathrm{Ca}$ and $\mathrm{Mg}$ in the soil and tomato leaf. Ayoola and Adeniyan (2006) also reported better performance of maize, cassava and melon under poultry manure + NPK fertilizer. The trend observed was NPK fertilizer + poultry manure $>$ NPK fertilizer $>$ poultry manure $>$ no fertilizer. It can be said that, the addition of NPK fertilizer to poultry manure aided mineralization of nutrients in poultry manure due to enhanced supply of nutrients, leading to better growth and yield. This study agrees with the findings of Makinde et al. (2001) who reported that the most satisfactory method of increasing maize yield was by judicious combination of organic wastes and inorganic fertilizer. Qian and Schoenau (2002), and Okwugwu and Alleh (2003) reported that high and sustained crop yield could be achieved with a judicious and balanced NPK fertilizer treatment combined with organic matter amendments. Ayoola and Adeniyan (2006) reported that nutrients from mineral fertilizers enhance the establishment of crops, while those from mineralization of organic manure promoted yield when both fertilizers were combined. The combined application of pig manure and NPK fertilizer also increased tomato fruit yield compared with pig manure or NPK fertilizer treatments alone (Giwa, 2004). Also, Adeniyan and Ojeniyi (2005) found that integrated application of poultry manure and NPK fertilizer increased maize yield compared with poultry manure or fertilizer applications alone.

\section{Conclusion}

Poultry manure improved soil nutrient status as indicated by increased soil organic carbon, total $\mathrm{N}$, available $\mathrm{P}$ and exchangeable $\mathrm{K}, \mathrm{Ca}$ and $\mathrm{Mg}$. The manure at $30 \mathrm{t} \mathrm{ha}^{-1}$ significantly increased growth and fruit yield when compared with the other levels. Results revealed that poultry manure is a suitable source of nutrients for improving soil fertility and yield of tomato especially if applied at $30 \mathrm{t} \mathrm{ha}^{-1}$ in the 
forest-savanna transition zone of southwest Nigeria. Compared with NPK fertilizer (300 $\left.\mathrm{kg} \mathrm{ha}{ }^{-1}\right)$, poultry manure improved the performance of tomato and its nutrient status. The combined application of poultry manure and NPK fertilizer increased the tomato fruit yield compared with the application of poultry manure or NPK fertilizer alone and is therefore recommended for a more sustainable tomato yield in the forest-savanna transition zone of Nigeria. In addition, the quantities of poultry manure and NPK fertilizer required would be reduced and tomato crop performance enhanced, therefore reducing the amount of money spent on chemical fertilizer.

\section{References}

Adediran, J. A., L. B. Taiwo and R. A. Sobulo. 2003. Comparative nutrient values of some solid organic wastes and their effect on tomato (Lycopersicon esculentum) yield. African Soils 33:99113.

Adeniyan, O. N. and S. O. Ojeniyi. 2005. Effect of poultry manure, NPK 15-1515 and combination of their reduced levels on maize growth and soil chemical properties. Nigerian Journal of Soil Science 15:34-41.

Agbede, T. M. 2006. Effect of tillage on soil properties and yam yield on an Alfisol in southwestern Nigeria. Soil Tillage Research 86:1-8.

Agbede, T. M., S. O. Ojeniyi and A. J. Adeyemo. 2008. Effect of poultry manure on soil physical and chemical properties, growth and grain yield of sorghum in southwest Nigeria. AmericanEurasian Joumal of Sustainable Agriculture 2 (1):72-77.

Agele, S. O. 2001. Growth and yield of tomato grown on degraded soil amended with organic wastes. Proceedings of the $35^{\text {th }}$ Conference of the Agricultural Society of Nigeria. Sept. 16-21. University of Agric. Abeokuta, Nigeria. pp. 151-154.

Akanni, D. I. 2005. Response of nutrient composition and yield components of tomato (Lycopersicon esculentum Mill) to livestock manure. Ph.D Thesis, Federal University of Technology, Akure, Nigeria. 120 pp.

Akanni, D. I. and S. O. Ojeniyi. 2007. Effect of different levels of poultry manure on soil physical properties, nutrient status, growth and yield of tomato (Lycopersicon esculentum). Research Journal of Agronomy 1: 1-4.

Akinrinde, E. A. and G. O. Obigbesan. 2000. Evaluation of the fertility status of selected soils for crop production in five ecological zones of Nigeria. Proceedings $26^{\text {th }}$ Annual Conference of Soil Science Society of Nigeria, Ibadan, Nigeria. pp. 279-288.

Ano, A. O. and J. A. Agwu. 2005. Effect of animal manures on selected soil chemical properties (1). Nigerian Journal of Soil Science 15: 14-19.

AOAC. 1990. Official Methods of Analysis. 15th ed. pp. 123-126. Association of Official Analytical Chemists, Washington DC. USA.

Ayoola, O. T. and O. N. Adeniyan. 2006. Influence of poultry manure and NPK fertilizer on yield and yield components of crops under different cropping systems in south west Nigeria. African Journal of Biotechnology 5 (15): 13861392.

Bremner, J. M. 1996. Nitrogen- Total. In: Sparks, D. I. (Ed.), pp. 1085-1121. Methods of Soil Analysis. Part 3. Chemical Methods. $2^{\text {nd }}$ edition, SSSA 
Book Series No. 5, ASA and SSSA, Madison, WI, USA.

Carter, M. R. 1993. Soil Sampling and Methods of Analysis. Canadian Society of Soil Science. Lewis Publishers, London. 823 pp.

Ewulo, B. S., S. O. Ojeniyi and D. A. Akanni. 2008. Effect of poultry manure on selected soil physical and chemical properties, growth, yield and nutrient status of tomato. African Journal of Agricultural Research 3 (9): 612-616.

Frank, K., D. Beegle and J. Denning. 1998. Phosphorus. In: Brown, J. R. (Ed.), pp. 21-26. Recommended Chemical Soil Test Procedures for the North Central Region, North Central Region Research Publication No. 221 (revised), Missouri Agric. Exp. Stn., Columbia, MO.

Giwa, D. D. 2004. Comparative effect of pig manure and NPK fertilizer on soil fertility and performance of tomato (Lycopersicon lycopersicum Mill.). M. Agric. Tech. Thesis, Federal University of Technology, Akure, Nigeria. 59 pp.

Hendershot, W. H. and H. Lalande. 1993. Ion exchange and exchangeable cations. In: Carter, M. R. (Ed.), vol. 19: 167176. Soil Sampling and Methods of Analysis. Canadian Society of Soil Science, Lewis Publishers, London.

Ibitoye, A. A. 2006. Laboratory Manual on Basic Soil Analysis. $2^{\text {nd }}$ ed. 82 pp. Foladave Publishing Company, Akure, Ondo State, Nigeria.

Makinde, E. A., A. A. Agboola and F. I. Oluwatoyinbo. 2001. The effects of organic and inorganic fertilizers on the growth and yield of maize in a maize/melon intercrop. Moor Journal of Agricultural Research 2: 15-20.
Mbah, C. N. 2006. Influence of organic wastes on plant growth parameters and nutrient uptake by maize (Zea mays L.). Nigerian Journal of Soil Science 16: 104-108.

Nelson, D. W. and L. E. Sommers. 1996. Total carbon, organic carbon and organic matter. In: Sparks, D. L. (Ed.), pp. 961-1010. Methods of Soil Analysis, Part 3. $2^{\text {nd }}$ edition, SSSA Book Series No. 5, ASA and SSSA, Madison, WI, USA.

Ojeniyi, S. O. 2000. Effect of goat manure on soil nutrients and okra yield in a rainforest area of Nigeria. Applied Tropical Agriculture 5: 20-23.

Okwugwu, M. T. and M. E. Alleh. 2003. The effect of organic and inorganic manures on the physico-chemical properties of the soil and yield of okra in Ekpoma, humid tropical rainforest of Nigeria. Proceedings $28^{\text {th }}$ Annual Conference of Soil Science Society of Nigeria, November $3^{\text {rd }}-7^{\text {th }}$, Umudike, Nigeria. Abstract p. 26.

Oladotun, A. O. 2002. Managing manure as a fertilizer; Saskatchewani, Agriculture, Food and Rural Revitalization. 5 pp.

Olasantan, F. O. 1991. Response of tomato and okra to nitrogen fertilizer in sole cropping and intercropping with cowpea. Journal of Horticultural Science 66: 191-199.

Osundare, B. 2004. Effects of fertilizer types and varying population on the performance of cocoyam. Moor Journal of Agricultural Research 5 (1): 7-12.

Qian, P. and J. J. Schoenau. 2002. Availability of nitrogen in solid manure amended with different $\mathrm{C}: \mathrm{N}$ ratios. Canadian Journal of Soil Science 82: 219-225. 
Emir. J. Food Agric. 2009. 21 (1): 10-20

http://cfa.uaeu.ac.ae/ejfa.shtml

Sheldrick, B. and C. Hand Wang. 1993. Particle-size distribution. In: M. R. Carter (Ed.), pp. 495-511. Soil Sampling and Methods of Analysis. Canadian Society of Soil Science, Lewis Publishers, Ann Arbor MI.

Steel, R. G. D. and J. H. Torrie. 1987. Principles and Procedures of Statistics. McGraw-Hill Book Co. Int. New York, $276 \mathrm{pp}$.
Stephenson, A. H., T. A. McCaskey and B. G. Ruffin. 1990. A survey of broiler litter composition and potential value as a nutrient resource. Biological Wastes 34: 1-9.

Tel, D. A. and M. Hagarty. 1984. Soil and Plant Analysis. IITA/University of Guelph. 277 pp. 